\title{
ST-Elevation Myocardial Infarction Networks and Logistics: Rural and Urban
}

\section{4}

\author{
Jithendra B. Somaratne, James T. Stewart, \\ Peter N. Ruygrok, and Mark W. Webster
}

\subsection{Introduction}

The facilities and expertise required for primary percutaneous coronary intervention (PPCI) of the infarct-related artery (IRA) in patients with ST-elevation myocardial infarction (STEMI) are only available at a limited number of hospitals. Fibrinolytic therapy, on the other hand, is more widely deliverable. This creates two distinct reperfusion choices: PPCI or a pharmacoinvasive strategy. The first option relies on immediate transfer to the closest PPCI-capable centre even if it means bypassing a closer non-PPCI centre. The second option is the "drip and ship" strategy. It involves delivery of fibrinolytic therapy by a non-PPCI facility with rapid transfer to a PPCIcapable centre.

In patients with ST-elevation myocardial infarction (STEMI), the primary therapeutic goal is the prompt restoration of coronary blood flow and myocardial perfusion. This limits the total ischaemic time and, thereby, the extent of the myocardial injury. In patients presenting within $12 \mathrm{~h}$ of symptom onset, PPCI undertaken within 120 min of diagnosis is the preferred reperfusion strategy. It leads to higher rates of IRA patency and thrombolysis in myocardial infarction (TIMI) 3 flow with lower rates of recurrent ischaemia, reinfarction, emergency repeat revascularisation, intracranial haemorrhage and death. This superiority over fibrinolytic therapy was observed when the delay to treatment was short and when patients were treated in high-volume centres by high-volume operators.

The choice of reperfusion strategy depends mainly on the time delays to treatment (Fig. 4.1), which can be categorised into patient- and system-related delays.

J. B. Somaratne $(\varangle) \cdot$ J. T. Stewart $\cdot$ P. N. Ruygrok $\cdot$ M. W. Webster

Green Lane Cardiovascular Service, Auckland City Hospital, Auckland, New Zealand

Auckland Mail Centre, Auckland, New Zealand

Green Lane Cardiovascular Service, Auckland City Hospital, Auckland, New Zealand

e-mail: jithendra.somaratne@adhb.govt.nz 


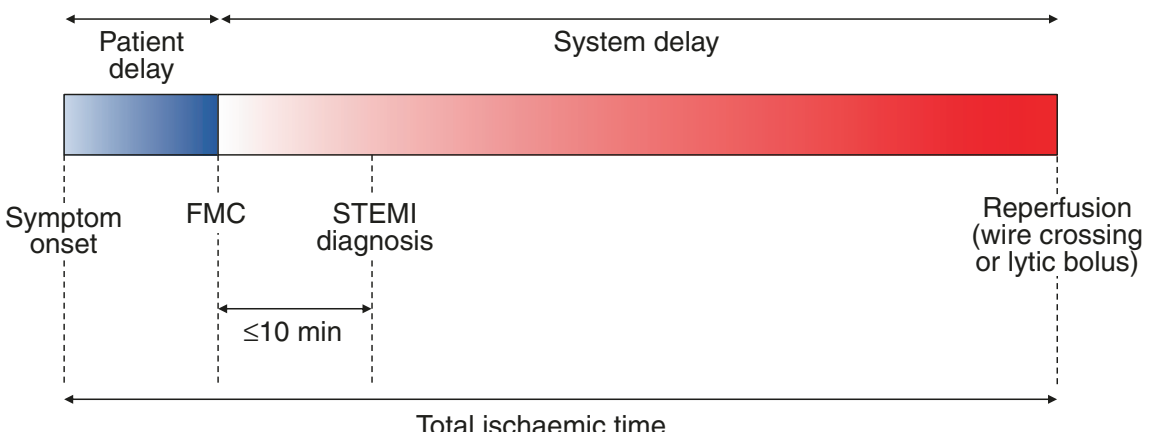

Fig. 4.1 Components of delay in management of patients with STEMI. FMC first medical contact, STEMI ST-elevation myocardial infarction

Patient delay accounts for the time taken from symptom onset to first medical contact (FMC). The widely accepted definition of FMC is the time of first assessment by a healthcare professional who is able to obtain and interpret a 12-lead electrocardiogram (ECG) and deliver initial interventions. System delay refers to the time from FMC to reperfusion. The time of reperfusion is defined as the commencement of fibrinolytic administration or, in the case of primary PCI, initial wire crossing. The benefit of fibrinolytic therapy has not been established in the context of patient delay $>12 \mathrm{~h}$. It could be considered if there is no possibility of PPCI and the patient has ongoing symptoms, particularly when there is a large area of myocardium at risk or there is haemodynamic instability. When the patient delay is between 12 and $48 \mathrm{~h}$, a routine PPCI strategy is recommended. There is no role for PCI to an occluded IRA when the patient delay is $>48 \mathrm{~h}$ and there are no ongoing symptoms. The task of minimising patient delay rests primarily with public health services. Steps to achieving the goal of reduced delay include increasing public awareness of common symptoms of myocardial ischaemia and the need for early recognition and activation of emergency medical services (EMS). In patients presenting within $12 \mathrm{~h}$ of symptom onset, anticipated system delay is the key determinant of reperfusion strategy (Fig. 4.2). If PPCI can be realistically achieved within 120 min of diagnosis, it is clearly preferred to fibrinolytic therapy. On the other hand, a pharmacoinvasive strategy should be adopted if the system delay associated with PPCI is expected to be $>120 \mathrm{~min}$.

The well-established relationship between system delay and mortality led to numerous efforts to accelerate the delivery of care. One of the most important advances in addressing system delay is the development of STEMI regional networks or systems of care, which are designed to enhance rapid recognition of STEMI and enable timely reperfusion therapy. Not all hospitals will have the capacity to provide a 24-h PPCI service in any given region. The reasons for this are twofold. Firstly, it is neither practical nor cost-effective to have multiple PPCI services operating within the same region. It leads to duplication of services, a reduction in efficiency and possible confusion of EMS regarding the most appropriate 


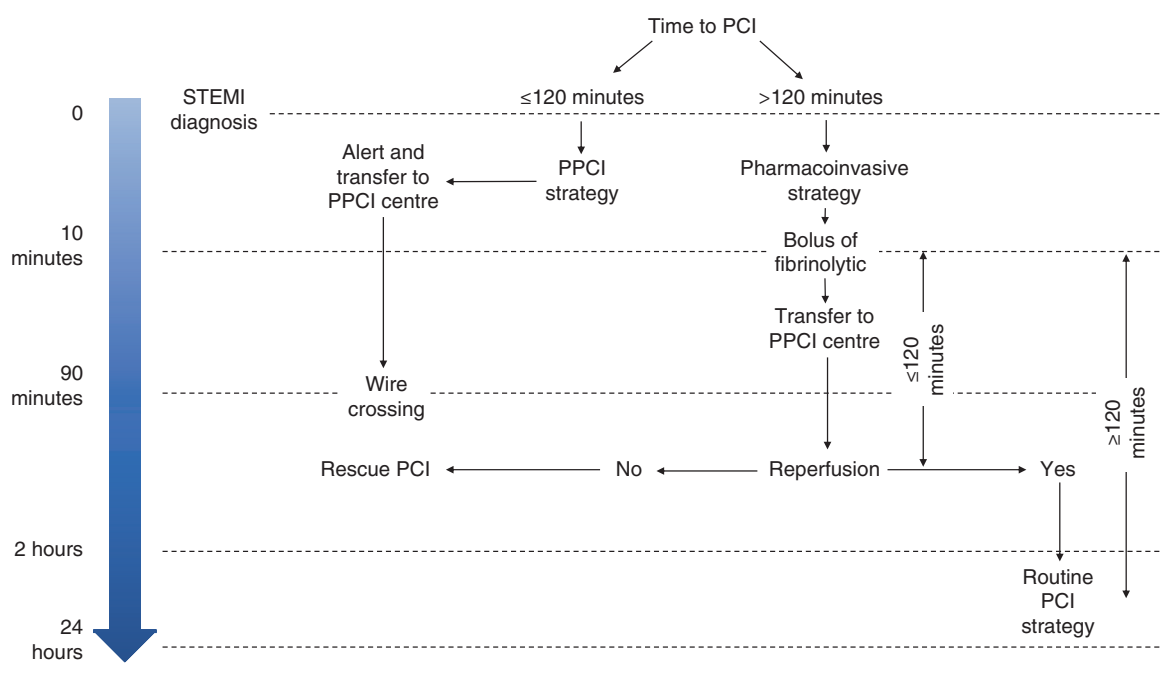

Fig. 4.2 Reperfusion strategy selection and timing. $P C I$ percutaneous coronary intervention, PPCI primary percutaneous coronary intervention, STEMI ST-elevation myocardial infarction

destination. In smaller hospitals, a 24-h PPCI roster may not be possible due to limited staffing. Secondly, there is a need to maintain high operator and institutional case volumes given the link between higher volume and better outcomes. These factors support a hub-and-spoke model of care. The hub is the PPCI-capable centre and the spokes are services that do not have PPCI capability. Each node of the network is linked by technology and by a prioritised and efficient EMS. It relies heavily on active multidisciplinary collaboration between cardiologists, emergency physicians and EMS. A well-organised regional network reduces system delay by using simple management algorithms that are locally developed, practical and acceptable to all stakeholders.

This chapter will describe the organisation and implementation of STEMI networks including the need for ongoing quality control initiatives. A case study is presented to highlight key ideas.

\subsection{Organisation of a Regional ST-Elevation Myocardial Infarction Network}

A successful STEMI network requires the close interaction of local services. They must be capable of responding to differences in patient presentation and geography (Fig. 4.3). For instance, some patients will self-present to a nearby primary care service or emergency department (ED), while others will call the EMS. The management of these patients will also depend on patient delay and their proximity to a PPCI service at the time they seek medical attention. The essential 


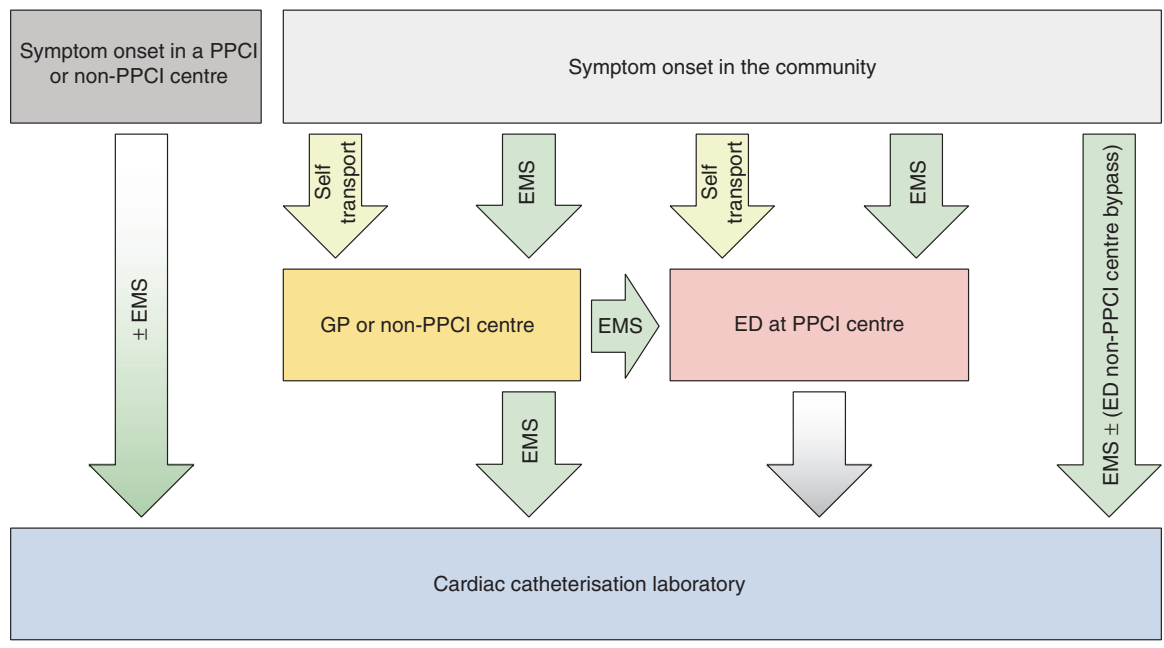

Fig. 4.3 Modes of patient presentation and transfer for primary percutaneous coronary intervention. $E D$ emergency department, $E M S$ emergency medical service, $G P$ general practitioner, $P P C I$ primary percutaneous coronary intervention

components of a network are the EMS, primary care facility or non-PPCI-capable centre, ED at a PPCI-capable centre and the cardiac catheterisation laboratory (CCL).

\subsection{Emergency Medical Service}

The EMS has an important role in early management patients with STEMI, which has evolved from patient transportation to include expedited initial diagnosis, triage and initiation of treatment. Depending upon the geography of the network, transfer of patients to a CCL may require a combination of transport modalities including road ambulances, helicopters and fixed-wing aeroplanes. The EMS may be staffed by suitably trained physicians, nurses or paramedics who are capable of basic and advanced life support.

To facilitate early diagnosis, EMS need to be equipped with a 12-lead ECG recorder and have trained personnel to acquire and interpret a trace correctly. This involves the correct application of leads, operation of the ECG recorder and recognition of different patterns consistent with STEMI or equivalent. Occasionally, paramedic interpretation of the ECG is not possible due to lack of training or experience. In this situation, the diagnosis may be based upon computer algorithm interpretation or physician interpretation of a transmitted ECG. While the computer algorithm output is easy to obtain, it is limited by a higher rate of erroneous interpretations. Wireless transmission and physician interpretation, on the other hand, have the highest level of interpretation accuracy and afford the opportunity for early physician direction of management. Wireless transmission requires transmitting 
and receiving technology at both ends as well as an available physician for interpretation at the receiving centre. This system is vulnerable to network and technologic failures.

Once the diagnosis has been established, the EMS needs a clear destination protocol to guide transportation to the most appropriate facility. For example, such protocols may authorise EMS units to bypass closer non-PPCI centres and take patients directly to a CCL.

Patients with STEMI are at higher risk of fatal arrhythmias such as ventricular tachycardia (VT) or ventricular fibrillation (VF). Accordingly, EMS providers must have the ability to undertake continuous cardiac rhythm monitoring and administer shocks with an external defibrillator.

Even though PPCI is often the preferred reperfusion strategy, rural paramedics, in particular, should be qualified to administer fibrinolytic therapy. If the delay associated with PPCI is expected to be $>120 \mathrm{~min}$, prehospital fibrinolysis is indicated.

\subsection{Primary Care Service or Non-primary Percutaneous Coronary Intervention Centre}

Sometimes patients with chest pain will transport themselves in a private vehicle to their local primary care service or the ED at a nearby non-PPCI hospital. These facilities are usually capable of recording and interpreting a 12-lead ECG. When a STEMI is diagnosed, the EMS should be activated and initial therapy commenced. This may include opioids and antithrombotic drugs including fibrinolysis. Yet, in comparison to a direct call to the EMS, self-presentation to a non-PPCI-capable facility is usually associated with a greater time delay to reperfusion. As such, patients with symptoms suggestive of a myocardial infarction should be discouraged from transporting themselves to a medical centre, by public health campaigns to raise awareness and understanding.

\subsection{Emergency Department at a Primary Percutaneous Coronary Intervention Centre}

The major tasks of an ED at a hospital that is PPCI-capable in patients with STEMI relate to diagnosis, initial therapy and immediate transfer to the CCL.

While there may be no doubt about the diagnosis in the majority of patients with STEMI brought into the ED by EMS, some may have an ambiguous clinical presentation. In this scenario, the ED staff assess the patient and clarify the diagnosis, in consultation with the cardiology service. In others, an alternate diagnosis may be found, and the ED physicians redirect patient care accordingly. Infrequently, the ED discover significant comorbidities (e.g. terminal cancer) that warrant de-escalation of care and adoption of a conservative approach. There are patients who present directly to the ED with chest pain. The ED team makes the initial diagnosis of STEMI for such patients. 
The two main initial therapies delivered in the ED are symptom relief and antithrombotic therapy. Pain relief is useful to minimise patient discomfort and reduce sympathetic activation. Intravenous opioids such as morphine are used for analgesia. Routine oxygen is no longer indicated although it should be administered in the context of hypoxia $\left(\mathrm{SpO}_{2}<90 \%\right)$. Patients are loaded with dual antiplatelet therapy in the form of aspirin and a $\mathrm{P} 2 \mathrm{Y}_{12}$ inhibitor (clopidogrel, prasugrel or ticagrelor). Depending on the local protocol, heparin or enoxaparin may also be given in the ED.

In the context of PPCI, the main role of the ED is to facilitate rapid patient transfer to the CCL. If the CCL is available and ready to receive, the patient should bypass ED altogether. However, this approach may not be achievable at all times. For instance, the CCL may be occupied by another patient or the on-call CCL team may not have arrived at the hospital. In this scenario, the ED performs the function of a holding bay until the patient can be transferred to the CCL. While the patient is waiting in ED, possible arterial access sites for coronary angiography should be prepared and initial blood samples sent to the laboratory for assessment. During this time, the patient is carefully observed with continuous cardiac monitoring and defibrillator nearby. The routine insertion of central venous or arterial access should be avoided as it often unnecessary and delays transfer to the CCL.

\subsection{Cardiac Catheterisation Laboratory}

The CCL is the final common destination for patients with STEMI within a regional network. Each region requires a CCL that is operational at all times even when the hospital is at maximum bed occupancy or the ED is closed due to overcapacity. It is recommended that each network has a single PPCI centre. There are a number of other more complex and less ideal models that may be employed. For instance, in some networks there are multiple PPCI centres or rotation of PPCI centre on a daily or weekly basis. The most recent European Society of Cardiology guidelines for the management of patients with STEMI recommend that any CCL that cannot offer 24-h service should be discouraged from offering PPCI. It is argued that the presence of daytime-only PPCI services may lead to confusion of EMS, affect the quality of intervention and increase system delay. The exception should be for patients admitted to those hospitals for a different reason that develop a STEMI during their hospital stay. However, this model is refuted by those belonging to networks in which alternate arrangements work well. A designated CCL for PPCI within a network should preferably be in a central geographic location within the region. Once the patient arrives on site, easy access to the CCL from the ambulance bay, helipad and ED enhances rapid transfer. Though patients with STEMI rarely require urgent surgical revascularisation, an on-site cardiac surgical facility is an advantage. The support provided by dedicated cardiac intensivists and cardiac anaesthetists at surgical centres is particularly useful when managing critically unwell patients in the CCL. The on-call CCL team usually comprises an interventional cardiologist, scrub nurse, circulating nurse, cardiac technologist and cardiac radiographer. 


\subsection{Implementation of a Regional ST-elevation Myocardial Infarction Network}

Over the last decade, there have been a number of initiatives around the world to develop and implement regional networks with the goal of improving the care of patients with STEMI in accordance with current guidelines. For example, in North America, the American Heart Association promoted Mission: Lifeline ${ }^{\circledR}$ and the American College of Cardiology ran the Door-to-Balloon (D2B): An Alliance for Quality campaign. Similarly, in Europe, the Stent for Life Initiative was a combined joint program by the European Society of Cardiology. To meet the tight guidelinebased target times for reperfusion, these initiatives have outlined a number of helpful approaches.

Prior to the designing and implementing a regional network, the Stent for Life Initiative recommends understanding existing local practice with regard to patients with STEMI. This investigation should seek to accurately establish the following statistics:

- Rates of STEMI

- Total number of patients with STEMI per annum

- Number and location of PPCI centres

- Ratio of PPCI centres to overall population

- Time taken patients to arrive at a PPCI centre

- Geographic constraints

- Modes of patient transportation

- Existing transportation protocols

- Equipment available on ambulances

- Level of training EMS personnel

- Proportion of the population served by a PPCI centre within a 90-min travel window

- Variation in the quality of service within the region

- Number of participating interventional cardiologists

- Number of interventional cardiologists per capita

- Reperfusion rates

- Mortality rate

Using the accumulated data, barriers to the delivery of timely reperfusion therapy can be identified. For instance, some of the commonly reported barriers include:

- Insufficient number of 24-h CCLs available for PPCI

- Lack of even geographic distribution of PPCI centres with overconcentration in urban areas

- Variation in the management of patients with STEMI throughout the region

- Delayed or inappropriate EMS response (such as non-adherence to the destination protocol) 
- Inadequately equipped or trained EMS

- Lack of EMS motivation to participate in network plans and protocols

- Commercial bias where there are too many CCL offering 24-h PPCI service

- Lack of quality control initiatives

- Poor public awareness of STEMI symptoms leading to delay in seeking medical attention

- Lack of a national registry

- Ineffectual communication and poor collaboration between key stakeholders within network

Once local barriers to timely care have been identified, the next step is to define realistic objectives and prioritise them. For example, the primary objective may be to initially develop a network focussed on a small region. Other possible objectives include setting up a quality control and feedback system or increasing public awareness about the symptoms warranting immediate medical attention. With these objectives in place, a strategic plan should be developed to realise each of them. Such planning has to involve all stakeholders in the networks including medical, transportation, industry and political partners.

Over the last decade, as it has become clear that treatment delays are associated with worse outcome in patients with STEMI, a number of strategies to minimise system delay were examined. Some of the successful approaches that reduce doorto-balloon time and thereby system delay are detailed below.

1. A careful and clear definition of geographic areas of responsibility reduces misunderstanding between each of the stakeholders in a regional network.

2. The use of a prehospital 12-lead ECG to activate the CCL while the patient is in route to the hospital allows time for the team to arrive and prepare to receive the patient in the CCL.

3. Limiting the number of stops on the patient journey from the community to the CCL reduces system delay. At times this will require the EMS to bypass nearby non-PPCI hospitals when a diagnosis of STEMI has been made. Likewise, on arrival at the appropriate hospital, bypassing the ED and heading straight to the CCL saves time. If a prehospital diagnosis was not made, the EMS should await the diagnosis in ED and immediately transfer the patient to a PPCI-capable hospital if the diagnosis is STEMI.

4. A discussion of the case and ECG findings by the ED physician with either a general cardiologist or interventional cardiologist prior to the activation of CCL incurs unnecessary delay. The ideal scenario is that the ED physician diagnoses the STEMI and activates the CCL immediately with consultation only when there is doubt.

5. Current laboratory and radiographic results should not be a prerequisite for activation of the CCL. Waiting for these results to be available leads to a further avoidable delay.

6. Using a single-call system to activate the CCL saves time. Typically, the call is to the hospital switchboard operator who then contacts each member of the 
CCL team. A return call can be made by the operator to the activating individual to confirm that the whole team has been notified. An even better option, if available, would be a simultaneous automatic activation system. This bypasses the operator and delivers the activation message simultaneously to each team member.

7. An expectation that the CCL team be available within 20 min of being paged and priority hospital parking during off-hours ensures that CCL staff-related delays are avoided. At some centres, the team is expected to be on site throughout the duration of their on-call period.

8. The initiation of patient transport from ED to CCL after a set time interval following activation rather than awaiting notification that the CCL team are ready was successful at reducing door-to-device time. Another approach is to transport the patient to the CCL when a minimum number of the CCL team arrive. Someone must always available to transport patients from ED to CCL. Whatever the approach adopted, a direct wireless phone line between an ED staff member and a CCL staff member facilitates communication and efficiency of patient transfer.

9. Each regional STEMI network is based on the collaboration of multiple organisations including hospitals, EMS and primary care facilities. The overall success of the network relies on the performance of each organisation. This, in turn, requires the support of senior management and an organisational culture that fosters and sustains change directed at minimising system delays. For those dealing with patients with STEMI directly, having a team-based approach from ambulance to device and a culture of continuous quality improvement is also very useful.

10. A regular multidisciplinary team review of local data with prompt feedback to all involved staff (i.e. EMS, ED and CCL) helps to identify deficiencies in the network and prompts continuous quality improvement initiatives. When significant delays occur in the care of individual patients, the use of a root cause analysis or similar approach helps to prevent recurrences.

\subsection{Quality Control}

Contemporary guidelines emphasise the importance of robust quality control measures within regional STEMI networks. A useful tool to monitor networks within a country is a national acute coronary syndrome registry. It can be used to ensure that simple quality of care indicators are met and maintained over time. The registry should record demographic data, relevant time points for the calculation of treatment delays, PPCI penetration, morbidity and mortality. Of these parameters, treatment delays are the most straightforward index of quality to audit. A simple comparison of practices and outcomes across different regions and within each region helps to identify outliers. If the continuously recorded quality of care indicators fall short of agreed benchmarks, then specific interventions addressing deficiencies should be devised and implemented 
to improve the performance of system. The data should be reviewed on a regular basis (e.g. quarterly). All major stakeholders in the network should receive feedback.

\subsection{Case Study}

The Northern Regional STEMI Network in New Zealand (NZ) includes both the Auckland and Northland regions. The Auckland region has a land area of $4894 \mathrm{~km}^{2}$ and a population of 1,657,200 in June 2017. This represents the highest population and second smallest land area of any region of NZ. It also has the largest economy in the country. The Northland region has land area of $13,789 \mathrm{~km}^{2}$ and a population of 175,500 in June 2017. The public hospitals and other health services in NZ are administered by district health boards (DHBs). There are 20 DHBs throughout the country and each is governed by a board of up to 11 members. They plan, manage, provide and purchase health services for the population of their district. The largely urban Auckland region has three DHBs: Auckland DHB (ADHB), Counties Manukau DHB (CMDHB) and Waitemata DHB (WDHB). The population of Northland is largely rural and is serviced by Northland DHB (NDHB). The Northern Regional STEMI Network is comprised of these four DHBs. Each is autonomous and has its own protocols for STEMI. The three urban DHBs (ADHB, CMDHB and WDHB) have CCLs that provide a PPCI service during standard working hours. However, ADHB has the only CCL in the network that offers a 24-h PPCI service. It is also the only one of the four to have on-site cardiac surgery. The NDHB does not have a CCL, and contracts ADHB for all cardiac catheterisation services. Patients with STEMI residing within the Northland region are transported to ADHB via helicopter. Within the Auckland region, PPCI within 120 min of STEMI diagnosis is feasible in the vast majority of patients. As a result, the default reperfusion strategy throughout the region is PPCI. The STEMI protocols for patients residing in the Northland region are necessarily more complex. The much smaller population of Northland is more widely spread out across a larger area. Patients self-presenting to or brought in by EMS to Whangarei Hospital, NDHB's base hospital, receive fibrinolytic therapy unless there is a contraindication. They are then transferred to ADHB for coronary angiography and PCI as indicated. At other smaller hospitals within the NDHB, the anticipated time delay to PPCI is considered and discussed with the on-call interventional cardiologist. If the delay to PPCI is expected to be over $120 \mathrm{~min}$, fibrinolytic therapy is administered, and the patient is transferred to ADHB soon after. Alternately, if the delay is calculated by the EMS to be less than 120 min, the local hospital is bypassed, and the patient is transferred urgently by helicopter to ADHB for PPCI. In the meantime, the on-call CCL team is activated to meet the patient in the CCL on arrival. All out-of-hours (5 p.m. to 8 a.m. and weekends; $73 \%$ of the week) PPCI for patients residing within the Auckland and Northland regions are undertaken in the CCL at ADHB. In a unique collaboration, the four interventional cardiologists from WDHB and the three interventional cardiologists from CMDHB join the four 
interventional cardiologists from ADHB in a network-wide on-call roster. Out-ofhours there is always one interventional cardiologist on call. The on-call CCL team includes a scrub nurse, circulating nurse, cardiac technologist and cardiac radiographer who are all employed by ADHB and work there during standard working hours. The on-call interventional cardiologist can be contacted on a dedicated free call number that is diverted to his/her mobile phone. The on-call interventional cardiologist or ED physician at ADHB is able to activate the CCL by contacting the operator at ADHB. In turn, the operator contacts each individual team member and often confirms with the interventional cardiologist that every team member has been contacted. There is an expectation that every member of the team, including the interventional cardiologist, should arrive within $20 \mathrm{~min}$ of the call-out. The individual data for all patients treated within the network are entered electronically into a national registry known as the All NZ Acute Coronary Syndrome Quality Improvement (ANZACS-QI) registry. The data concerning patients with STEMI are analysed and presented to stakeholders on a quarterly basis. In addition, regular network meetings every 6 weeks provide a forum for the discussion of specific concerns as they arise.

\subsection{Summary}

The management of patients with STEMI is time critical. The choice of reperfusion strategy is contingent upon patient and system delay. Primary PCI is preferred over a pharmacoinvasive approach when the anticipated system delay is short. While public health initiatives help to reduce patient delay, system delay is minimised by well-designed effective regional networks or systems of care. These systems rely on the collaboration of a number of local services such as the EMS, non-PPCI facilities and PPCI hospitals. They lead to better patient outcomes by reducing the time to diagnosis and treatment. Within networks, continuous quality control initiatives with regular feedback to all stakeholders are vital to maintain standards and improve the service.

\section{Further Readings}

Bradley EH, Roumanis SA, Radford MJ, Webster TR, McNamara RL, Mattera JA, et al. Achieving door-to-balloon times that meet quality guidelines: how do successful hospitals do It? J Am Coll Cardiol. 2005;46(7):1236-41.

Bradley EH, Herrin J, Wang Y, Barton BA, Webster TR, Mattera JA, et al. Strategies for reducing the door-to-balloon time in acute myocardial infarction. $\mathrm{N}$ Engl $\mathrm{J}$ Med. 2006;355(22):2308-20.

Bradley EH, Nallamothu BK, Curtis JP, Webster TR, Magid DJ, Granger CB, et al. Summary of evidence regarding hospital strategies to reduce door-to-balloon times for patients with ST-segment elevation myocardial infarction undergoing primary percutaneous coronary intervention. Crit Pathw Cardiol. 2007;6(3):91-7.

Danchin N. Systems of care for ST-segment elevation myocardial infarction: impact of different models on clinical outcomes. JACC Cardiovasc Interv. 2009;2(10):901-8. 
Holmes DR, Bell MR, Gersh BJ, Rihal CS, Haro LH, Bjerke CM, et al. Systems of care to improve timeliness of reperfusion therapy for ST-segment elevation myocardial infarction during off hours: the Mayo Clinic STEMI protocol. JACC Cardiovasc Interv. 2008;1(1):88-96.

Ibanez B, James S, Agewall S, Antunes MJ, Bucciarelli-Ducci C, Bueno H, et al. ESC guidelines for the management of acute myocardial infarction in patients presenting with ST-segment elevationThe task force for the management of acute myocardial infarction in patients presenting with ST-segment elevation of the European Society of Cardiology (ESC). Eur Heart J. 2017;33(20):2569-619.

Knot J, Widimsky P, Wijns W, Stenestrand U, Kristensen SD, Van THA, et al. How to set up an effective national primary angioplasty network: lessons learned from five European countries. EuroIntervention. 2009;5(3):299, 301-9.

Nestler DM, Noheria A, Haro LH, Stead LG, Decker WW, Scanlan-Hanson LN, et al. Sustaining improvement in door-to-balloon time over 4 years. Circ Cardiovasc Qual Outcomes. 2009;2(5):508.

O'Gara PT, Kushner FG, Ascheim DD, Casey DE, Chung MK, de Lemos JA, et al. 2013 ACCF/ AHA guideline for the management of ST-elevation myocardial infarction. A report of the American College of Cardiology Foundation/American Heart Association task force on practice guidelines. Circulation. 2013;127(4):e362-425.

Open Access This chapter is licensed under the terms of the Creative Commons Attribution 4.0 International License (http://creativecommons.org/licenses/by/4.0/), which permits use, sharing, adaptation, distribution and reproduction in any medium or format, as long as you give appropriate credit to the original author(s) and the source, provide a link to the Creative Commons license and indicate if changes were made.

The images or other third party material in this chapter are included in the chapter's Creative Commons license, unless indicated otherwise in a credit line to the material. If material is not included in the chapter's Creative Commons license and your intended use is not permitted by statutory regulation or exceeds the permitted use, you will need to obtain permission directly from the copyright holder.

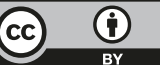

